\title{
Experimental techniques for aberration retrieval with through-focus intensity images
}

\author{
S. F. Pereira ${ }^{a}$, A. Wiegmann ${ }^{b}$, N. Kumar ${ }^{a}$, A. da Costa Assafrao ${ }^{a}$, A. Polo $^{a}$, L. Wei $^{a}$, and S. \\ van Haver $^{a}$ \\ a Optics Research Group, Department of Imaging Science and Technology, \\ Faculty of Applied Sciences, Delft University of Technology, \\ Lorentzweg 1, NL-2628 CJ Delft, The Netherlands \\ ${ }^{b}$ Physikalisch-Technische Bundesanstalt, \\ Bundesallee 100, 38116 Braunschweig, Germany
}

\begin{abstract}
Measurement techniques to determine the aberration of an optical system, by obtaining through-focus intensity images that are produced when the object is a point source at infinity, are shown. The analysis of the aberrations is made using the extended version of the Nijboer-Zernike diffraction theory. This theory provides a semi analytical solution of the Debye diffraction integral and thus a direct relation between the intensity distribution of the field at the focal region and the exit pupil of the optical system.
\end{abstract}

Keywords: Diffraction, point-spread function, Debye integral, phase retrieval, aberrations

\section{INTRODUCTION}

Knowledge of the aberrations of an optical system allows us to predict its quality, and it represents essential information in the assessment of high quality imaging systems as for example, projection systems in optical lithography and microscopy. The most common approach in obtaining the aberration coefficients is by applying interferometric tools such as Michelson, Fizeau or Twyman-Green interferometers. ${ }^{1}$ These techniques are already known for decades, and precise tools are available commercially. More recently, with the improvement of the quality and resolution of Shack-Hartmann wavefront sensors, it has been shown that these sensors can also be used for characterization of optical elements. ${ }^{2,3}$ However, both techniques have some drawbacks; on one hand the requirement of special setups and environmental conditions and on the other hand, reference calibration and signal-to-noise ratio can be an important issue.

In this paper, we consider another approach to aberration retrieval, namely a method where only intensity measurements are needed. The measurement that enables the determination of aberrations consists in collecting through-focus intensity images that are produced by the imaging system under test when the object is (approximately) a point source at infinity. Due to aberrations, the intensity distribution in the various transversal planes around the focal plane is not the same as in the aberration-free case. Although the knowledge of the intensity planes does not directly give information on the phase of the field, it has been shown that the phase function in the exit pupil can be retrieved from intensity data. This can be achieved when one has enough data diversity available and in some cases, a-priori information. ${ }^{4-6}$ In particular, the extended Nijboer-Zernike (ENZ) theory is a powerful framework that allows us to obtain a semi-analytical solution for the Debye diffraction integral which describes the relation between the field in focus and the complex exit pupil of the optical system. By using this semi-analytical approach, one has not only insight to the problem but also high retrieval accuracy can be achieved. ${ }^{6,7}$

However, on the experimental side, in order to acquire intensity measurements containing enough information to allow aberration reconstruction, enough resolution in both the lateral and the longitudinal directions in the focal region is required. To this goal, we have implemented two experimental methods that are described in detail in this paper; in the first one, the intensity planes are obtained by direct measurement at the focal region

Further author information: Send correspondence to S.F.Pereira(@tudelft.nl)

Optical Design and Testing V, edited by Yongtian Wang, Chunlei Du, Hong Hua, Kimio Tatsuno, H. Paul Urbach, Proc. of SPIE Vol. 8557, 855706 • (C) 2012 SPIE · CCC code: 0277-786/12/\$18 · doi: 10.1117/12.2000376 
by using a high resolution scanning probe, and in the second method, the focal region is re-imaged with a given magnification on a CCD camera.

The paper is organized as follows: in Section 2 we present a brief explanation of the theoretical approach, in Section 3 we show details of the experimental setups and the experimental results, and in Section 4 we present discussions followed by conclusions in Section 5 .

\section{THEORETICAL APPROACH}

In this section, some theoretical aspects about the Extended Nijboer Zernike method are given. ${ }^{7}$ This method allows us to obtain a semi-analytic solution of the Debye diffraction integral for an imaging system with a pupil function characterized by phase deviations $\Phi$ (aberration) and amplitude defects $A(\rho, \theta)$. The transmission function of the optical system is then mapped into a single complex function given by

$$
g(\rho, \theta)=A(\rho, \theta) \exp \{i \Phi(\rho, \theta)\},
$$

with $\rho$ and $\theta$ the normalized radial and azimuthal coordinate on the exit pupil sphere, $\Phi(\rho, \theta)$ the phase deviation and $A(\rho, \theta)$ the amplitude transmission function. This complex pupil function is expanded into a Zernike expansion with complex coefficients $\beta_{n}^{m}$ according to ${ }^{6}$

$$
A(\rho, \theta) \exp \{i \Phi(\rho, \theta)\}=\sum_{n, m} \beta_{n}^{m} R_{n}^{|m|}(\rho) \exp (i m \theta),
$$

with $R_{n}^{m}(\rho)$ being the radial part of the Zernike polynomial of degree $n$ and azimuthal order $|m|$, for $n \geq 0$ and $n-|m| \geq 0$ and even. Note that the azimuthal parameter $m$ can take both positive and negative integer values. The ENZ diffraction theory provides a solution of the Debye diffraction integral in terms of a series expansion with respect to the defocus parameter and the radial image coordinate. The azimuthal dependence of a general defocused intensity pattern is given by means of a harmonic expansion with respect to the azimuthal image coordinate. The Debye formulation of the diffraction integral is sufficiently accurate if the distance from exit pupil to image region tends to infinity (telecentric imaging condition). In general, objective lenses such as the ones considered in our experiments satisfy this requirement. For the interested reader, the theoretical details of the extended Nijboer-Zernike diffraction theory are found in Ref. ${ }^{6-9}$ The measurement data required for reconstruction is taken from the three-dimensional point-spread function on the image side of the optical system. In order to retrieve the phase function in the exit pupil of the optical system from these intensity measurements, one can proceed in several ways. ${ }^{14-16}$ In the case of the ENZ, a linear system of equations is devised, relating the measured intensity directly to the Zernike coefficients describing the transmission function. ${ }^{9,12,13,17}$ In the small aberration limit, solving this linear system directly yields the coefficient you are looking for. When aberrations are larger, the solution is not exact due to the error introduced by linearizing the theoretical expression of the 3D point spread function. However, also in this case, ENZ can provide accurate results via an iterative scheme. ${ }^{9-11}$

\section{MEASUREMENT APPROACH}

\subsection{Direct measurement of the field in the focal region using a subwavelength scanning probe}

In this section, we show the details of the experimental setup and results obtained with the direct measurement of the through-focus field intensities. The general scheme of the experiment is shown in Fig. 1. A diode laser operating at the wavelength $405 \mathrm{~nm}$ is focused onto a single mode optical fiber and collimated at the output with an off-axis parabolic mirror. This collimated beam is incident on a DVD lens with numerical aperture NA=0.65; that is designed to be used with an aberration correction glass plate of $600 \mu \mathrm{m}$. The lens is mounted on a $\mathrm{x}-\mathrm{y}-\mathrm{z}$ piezo stage. The aberration correction glass plate is placed within the working distance of the DVD lens in a way that the focal region lies outside the glass plate. The intensity in the $\mathrm{x}-\mathrm{y}$ plane of the focused field after the glass plate at a particular z position is measured point-by-point by coupling the light onto a pyramid-type near field probe with an aperture of $90 \mathrm{~nm}$. This subwavelength probe is placed on the focal plane of the collection lens (see Fig. 1). The light that is caught by the collection lens is focused onto a multimode fiber and detected 
by a photomultiplier. Each through-focus plane ( $\mathrm{x}-\mathrm{y}$ plane) is obtained by scanning an area of a few $\mu \mathrm{m}^{2}$ using the scanning table. Once one $\mathrm{x}-\mathrm{y}$ scan is obtained, the tip is moved up(down)wards to a new z position where the $\mathrm{x}-\mathrm{y}$ scan is repeated. In order to show how aberrations affect the through-focus intensity planes, we have also obtained the same intensity data but without the $600 \mu \mathrm{m}$ correction plate. A set of through-focus planes without the correction plate is shown in Fig. 2. In the bottom of the figure we also plot the on-axis intensity of the field corresponding to various through-focus planes. According to Ref. ${ }^{20}$ since the DVD lens has been designed to work with the correction plate, the absence of the same will result in spherical aberrations, which in our case, the best fit corresponded to the Seidel coefficients $W_{40}=12.76$ and $W_{60}=0.0062$ (in wavelength units, with $\lambda=400 \mathrm{~nm}$ ) for the case without the correction plate. This aberration is clearly seen in the intensity on axis as plotted in Fig. 2, bottom left. In this plot the red points are the experimental data, while the solid black line is the theoretical fit based on the expected spherical aberration. For comparison with the ideal case, we also plot the aberration-free profile (bottom figure, right side), calculated theoretically.

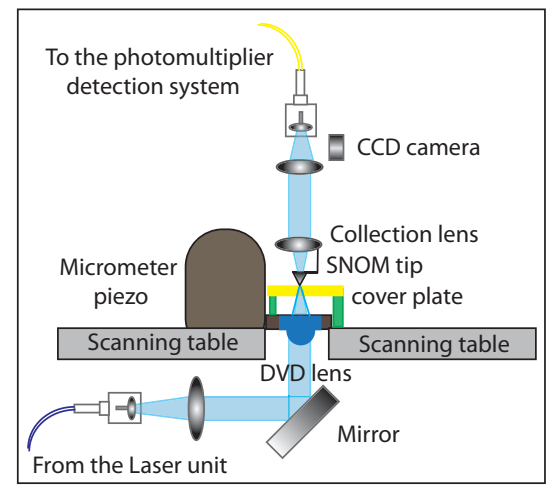

Figure 1: Lightpath of the direct method using a subwavelength probe.

\subsection{Measurement of the field in the focal plane using magnification}

In this section, we show the details of the experimental setup and results obtained with the measurement of the through-focus field intensities using magnification and a CCD camera. In Fig. 3 we show the main optical path of the experimental setup. As light source, we used an intensity stabilized He Ne laser coupled in a single mode optical fiber. As it can be seen in Fig. 3, the collimated beam from the optical fiber illuminates the test lens and the focal region is imaged onto a CCD camera by an imaging system containing a high numerical aperture objective and a tube lens. The lateral dimension of the input field at the test lens is large enough so that an approximately uniform field is obtained at the test lens. The degree of collimation of the beam has been measured with a Shack-Hartmann sensor resulting in a rms about $\lambda / 100$ on a beam size of $8 \mathrm{~mm}$ (diameter of the objective). In order to obtain the through-focus planes, the test lens is mounted on a $\mathrm{x}-\mathrm{z}$ translation stage, where the $\mathrm{z}$ direction corresponds to the optical axis and the $\mathrm{x}$ direction (i.e., perpendicular to the $\mathrm{z}$ direction) to the lateral displacement of the lens. A displacement interferometer has been used to measure the position of the mechanically driven $\mathrm{z}$ translation stage so that the position of each of the through-focus planes can be determined with high precision.

Further remarks about this setup are that since the data is not taken directly in the focal region (as was the case in Ref. ${ }^{17}$ and in the direct method shown in Section 3.1), the knowledge of the exact magnification factor is also needed. Experimentally, this has been done by moving the test lens in the $\mathrm{x}$ direction with the calibrated piezo stage by a certain known value and recording the corresponding shift of the spot at the CCD camera. The magnification of the setup is 50.4, which gives enough resolution for detection of the field intensity at the focal region given that the pixel size of the CCD camera is $3.75 \mu \mathrm{m}$. Furthermore, it is also important to calibrate the optics that is used for the magnification (i.e., imaging objective and tube lens), since the aberrations of these two lenses will be included in the field intensity distribution measured at the CCD camera. Finally, it is also worth mentioning the response of the CCD camera. Most commercial cameras have adaptable gain that is automatically set in order to avoid saturation of the image. In our case, this cannot be used, since the relative 
intensity from the various through-focus images should be kept. The linearity of the response of the CCD camera over the entire dynamic range should be guaranteed.
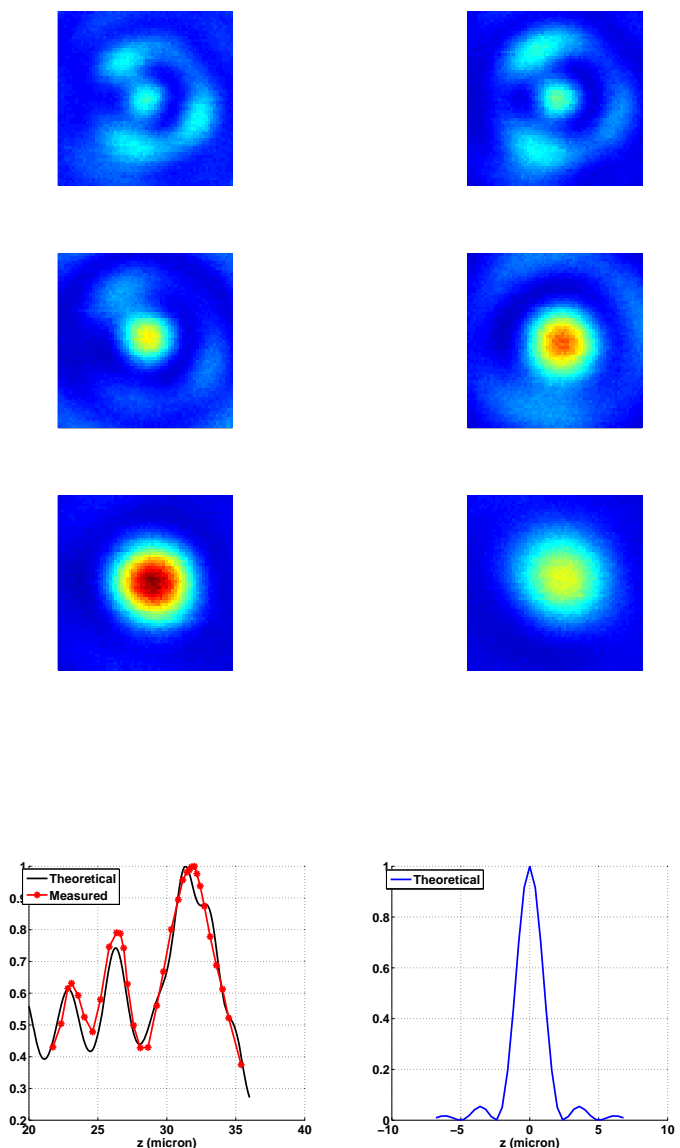

Figure 2: Set of 6 through-focus intensity data of a DVD lens without aberration correction plate obtained with the direct method. The scan area is $2 \times 2$ microns. (Bottom left): on-axis intensity of the focal region without aberration correction plate (red), theoretical fit with spherical aberrations (solid black line). (Bottom right): theoretical on-axis intensity of the focal region with correction aberration plate.

With this experimental configuration, we have obtained the through-focus intensity data of an objective of numerical aperture $\mathrm{NA}=0.4$, with diameter of $8 \mathrm{~mm}$. A set of six through-focus intensity field distributions is shown in Fig. 4. During the experiments, the dark field is also obtained in order to compensate for background light. As it has been done in the previous section, in order to see the effect of aberrations in the intensity distribution of the through-focus planes, we also obtained the same data as shown in Fig. 4 but with an extra aberration plate placed before the objective ( $1 \lambda$ spherical aberration on a diameter of $12.5 \mathrm{~mm})$. The on-axis intensities of these two data sets (with and without aberration plate) can be seen in the plots at the bottom of the Fig. 4.

Using the data with and without aberration plate, the phase retrieval method based on the extended NijboerZernike theory has been used to reconstruct the phase of the wavefront of the aberration plate. In this case, the aberrations of the optics and the systematic errors present in the system are eliminated. The reconstruction results have been compared with independent measurements using an interferometric setup (Fizeau interferometer Zeiss Direct $100^{18}$ ), resulting in a rms difference of the order of $7 \mathrm{~nm} .{ }^{19}$ This result confirms that through-focus intensity method is potentially a good alternative to present aberration retrieval systems. 


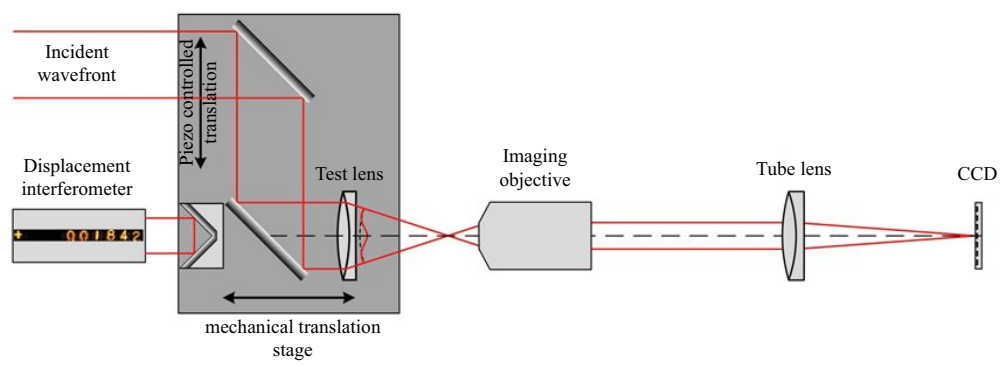

Figure 3: Scheme of the experimental setup to measure the field in the focal region with magnification
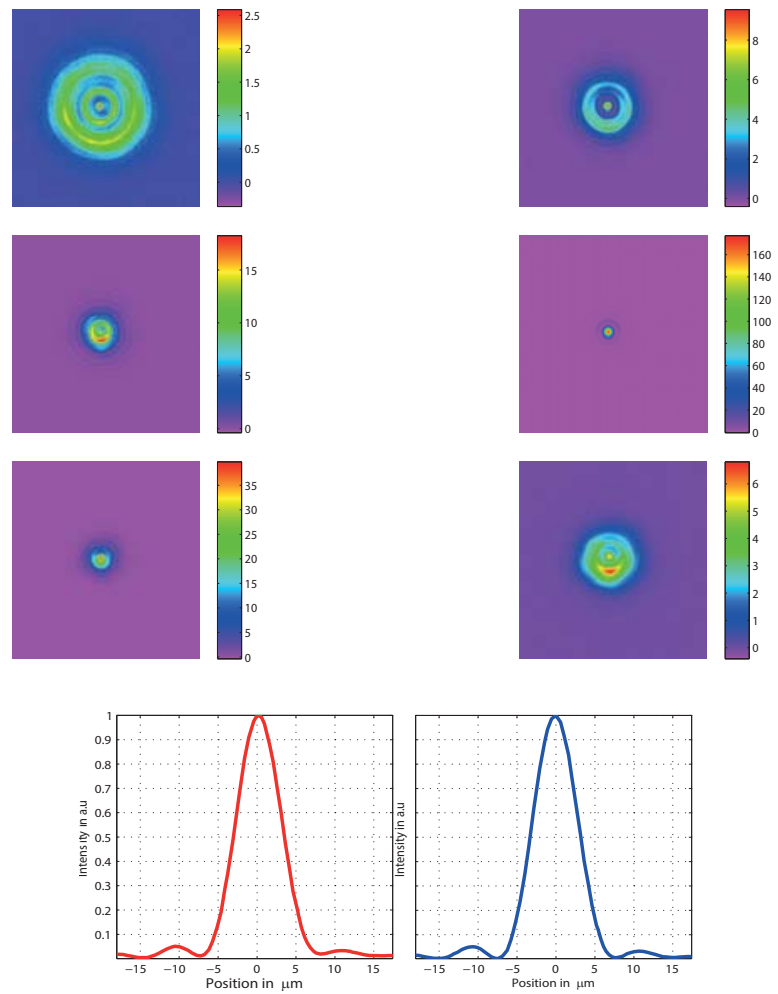

Figure 4: Set of through-focus intensity data obtained with the magnification method using a CCD camera of a test lens with numerical aperture 0.4. (Bottom left): on-axis intensity when the aberration plate is inserted before the test lens. (Bottom right): on-axis intensity without the aberration plate. Note that the difference between the two plots lies mostly in the values of the first minima.

\section{DISCUSSIONS}

In this paper we show two experimental approaches to achieve through-focus intensity images of focused fields with high lateral and longitudinal resolution. Both approaches have advantages and disadvantages, as listed below:

Direct method:

- the field intensity is measured at the focal region; thus no magnification system is needed. This implies that with this method only the $\mathrm{x}-\mathrm{y}-\mathrm{z}$ translations should be independently calibrated;

- the setup involves $\mathrm{x}-\mathrm{y}$ scanning to obtain each $\mathrm{x}-\mathrm{y}$ plane; this is generally slower than in the magnification method where one $\mathrm{x}-\mathrm{y}$ plane is obtained in one shot at the CCD camera;

- in general, it is assumed that the measured intensity at the photomultiplier due to a single point in the x-y plane is proportional to the intensity at the at the probe. This is not exactly true if different polarizations 
are present in the focal region. For example, the longitudinally polarized field couples onto the probe with different efficiency as compared to the transversally polarized field. ${ }^{21}$ This issue should be taken into account when high numerical apertures are involved.

Magnification method:

- as discussed in the last section, the measurement of the field in the focal region has contributions both of the test lens and the magnification optics. In order to extract the aberrations of the test lens only, one should make an independent measurement of the aberrations of the imaging and tube lens (see Fig. 3). This can be done in the setup by adding a subwavelength pinhole at the focal plane of the imaging objective and measuring the through-focus planes. In this case, the measured point spread function will contain the aberrations of the magnification system.

- this setup is simpler than the previous one and can be adapted in many existing optical setups (such as an imaging system), so that the aberrations of the entire system could be measured in-situ.

\section{CONCLUSIONS}

In conclusion, we have shown two experimental methods that have been used to obtain through-focus intensity field distributions with high lateral and longitudinal resolution, namely a direct method based on scanning field subwavelength probe, and a method where the focused area is magnified and recorded with a CCD camera. Using these setups, we show measurements of the intensity planes of two test lenses of numerical aperture of 0.65 and 0.4 , respectively. The data has been used to obtain information about the aberrations of the test lenses, and using the extended Nijboer-Zernike formalism, phase retrieval has been achieved and compared with interferometric measurements giving a satisfactory result. A comparison between the two methods has been made. The methods presented have advantages over interferometric ones, since it can be done in-situ and no reference surfaces are needed. Important experimental issues consist of proper calibration of the $\mathrm{x}$-y-z-translation axis, magnification system and response of the CCD camera.

\section{ACKNOWLEDGMENTS}

The authors acknowledges the financial support of the Dutch Ministry of Economic Affairs and the Provinces of Noord-Brabant and Limburg in the frame of the Pieken in de Delta program, the NanoNextNL of the Government of the Netherlands and 130 partners, and the FP-7 European project SURPASS (proj. n. 224226).

\section{REFERENCES}

[1] W. H. Steel, Interferometry, Cambridge University Press, London, 1967.

[2] J. Pfund, N. Lindlein, and J. Schwider, "Absolute sphericity measurement: a comparative study of the use of interferometry and a Shack-Hartmann sensor", Opt. Lett. 23, 742-744 (1998).

[3] J. A. Koch et al., "Experimental comparison of a Shack-Hartmann sensor and a phase-shifting interferometer for large optics metrology applications", Appl. Opt. 39, 4540-4556 (2000).

[4] R. A. Gonsalves, "Phase retrieval and diversity in adaptive optics", Opt. Engin. 21, 829-832 (1982).

[5] O. El Gawhary, private communication.

[6] J. J. M. Braat, S. van Haver, A. J. E. M. Janssen, P. Dirksen, "Assessment of optical systems by means of point-spread functions", in [Progress in Optics], E. Wolf, ed., 51, chap. 6, 349-468, Elsevier B. V. (2008).

[7] A. J. E. M. Janssen, "Extended Nijboer-Zernike approach for the computation of optical point spread functions", J. of the Opt. Soc. Am. 19, 849-857 (2002).

[8] A. J. E. M. Janssen, "New analytic results for the Zernike circle polynomials from a basic result in the Extended Nijboer-Zernike diffraction theory", J. of the Eur. Opt. Soc. Rap. Public. 6, 11028 (2011).

[9] S. van Haver, "The Extended Nijboer-Zernike diffraction theory and its applications", Ph.D. thesis, Delft University of Technology, 2010.

[10] A. Asensio Ramos, A. Lopez Ariste, "Image reconstruction with analytical point spread function", Astron. and Astrophys. 418, A6 (2010). 
[11] L. Liu, L. Wang, J. Wang, H. Meng, "A drie dimensional point spread function for phase retrieval and convolution", Opt. Expr. 20, 14, 15392-15405 (2012).

[12] P. Dirksen, J. J. M. Braat, A. J. E. M. Janssen, and A. Leeuwstein, "Aberration retrieval for high NA optical systems using the Extended Nijboer-Zernike theory", in [Proc SPIE], 5754, 262-273 (May 2005).

[13] S. van Haver, J. J. M. Braat, P. Dirksen, A. J. E. M. Janssen, "High-NA aberration retrieval with the extended Nijboer-Zernike vector diffraction theory", J. of the Europ. Opt. Soc. Rap. Public, 1, 06004 (2006), and 2, 07011e (2007).

[14] J. R. Fienup, "Phase retrieval algorithms: a comparison", Appl. Opt. 21, 2758-2769 (1982).

[15] R. W. Gerschberg and W. O. Saxton, "A practical algorithm for the determination of the phase from image and diffraction pictures", Optik 35, 237-246 (1972).

[16] J. Miao, D. Sayre, H. N. Chapman, "Phase retrieval from the magnitude of the Fourier transforms of nonperiodic objects", Opt. Lett. 28, 801-803 (2003).

[17] P. Dirksen, J. J. M. Braat, A. J. E. M. Janssen, "Estimating resist parameters in optical lithography using the Extended Nijboer-Zernike theory", J. Microlith., Microfabr., Microsys. 5, 1-11 (2006).

[18] M. F. Kruechel, "New Zeiss inteferometer", [Proc SPIE], 1332, 655 (1991).

[19] A. Wiegmann, S. van Haver, N. Kumar, and S. F. Pereira, "Experimental validation of the extended Nijboer-Zernike (ENZ) based aberration retrieval method for microscopic objectives", DGaO Proceedings, ISSN: 1664-8436 (2012).

[20] J. J. M. Braat, "Analytical expressions for the wave-front aberration coefficients of a tilted plane parallel plate", Appl. Opt. 36, 8459-8466 (1997).

[21] B. Jia, X. Gan, M. Gu, "Direct observation of a pure focused evanescent field of high numerical aperture objective lens by scanning near-field optical microscopy", Appl. Phys. Lett. 86, 131110-1:3 (2005). 